\title{
Rapid Eye Movement (REM) rebound on initial exposure to CPAP therapy: a systematic review and meta-analysis
}

\author{
Gaurav Nigam", Macario Camacho ${ }^{2}$ and Muhammad Riaz ${ }^{3}$
}

\begin{abstract}
Objective: Rapid Eye Movement (REM) rebound is a polysomnographic phenomenon where a substantial increase in REM sleep is noted in patients with untreated obstructive sleep apnea (OSA) when first undergoing continuous positive airway pressure (CPAP) titration. The objectives of this study are to determine: 1) the percentage of patients experiencing REM rebound during CPAP titrations, 2) to quantify the relative increase in REM sleep duration and 3) to identify if there are patient variables associated with REM rebound.
\end{abstract}

Methods: Four databases (including PubMed/Medline) were systematically searched through March 12, 2017.

Results: Four hundred sixty-seven articles were screened, 58 were reviewed in full-text form and 14 studies met the criteria for inclusion in this review. Eleven of the fourteen studies noted a statistically significant increase in amount of REM sleep during the titration night, compared to baseline sleep study. Pre- and post-CPAP REM sleep duration percentage means \pm standard deviations $(M \pm S D)$ in 1119 patients increased from $13.8 \pm 8.2 \%$ to $20.0 \pm 10.1 \%$; random effects modeling demonstrated a mean difference of 7.86 (\%) [95\% Cl 5.01, 10.70], p-value $<0.00001$, corresponding to a 57\% relative increase in REM sleep duration. The standardized mean difference (SMD) is 0.90 [95\% Cl 0.59, 1.22], representing a large magnitude of effect.

Conclusions: In studies reporting REM rebound, the REM sleep duration increased by 57\% during the first CPAP titration night compared to the baseline sleep study. The prevalence of REM rebound varied between 23 and 46\%. A low amount of REM sleep on the diagnostic PSG predicted REM rebound.

Keywords: REM rebound, Continuous positive airway pressure, Cortical arousal, Adherence, Obstructive sleep apnea, Sleep hypnogram

\section{Background}

The hypnogram obtained during a sleep study in a patient with untreated obstructive sleep apnea (OSA) has certain distinct features. The majority of them have fragmented sleep architecture with limited amounts of Rapid Eye Movement (REM) and slow wave sleep. Historically, the sleep fragmentation has been attributed to the preponderance of arousals that are temporally associated with respiratory events in patients with untreated OSA (Remmers et al. 1978). Early experimental studies of selective sleep deprivation have demonstrated that stage REM sleep is highly susceptible to eradication

\footnotetext{
* Correspondence: dr.nigamgaurav@gmail.com

'Clay County Hospital, 911 Stacy Burk Drive, Flora, IL 62839, USA

Full list of author information is available at the end of the article
}

by repetitive arousals, more so than stage slow wave sleep (Agnew et al. 1967). This might suggest that REM sleep is more frequently abolished by untreated OSA, as compared to slow wave sleep.

When first exposed to continuous positive airway pressure (CPAP), certain favorable changes occur in the sleep architecture. CPAP therapy leads to a reduction in the arousal index, sleep stage shifts and non-rapid eye movement (NREM) stage 1 (Loredo et al. 2006). Consequently, sleep becomes more consolidated with an increase in the duration of REM sleep compared to the diagnostic study. REM rebound (Brillante et al. 2012; Koo et al. 2012; Kushida et al. 2011; Yaegashi et al. 2009; Osuna et al. 2008; Drake et al. 2003; Verma et al. 2001; Randerath et al. 2001; Parrino et al. 2000; Yamashiro and 
Kryger 1995; Lamphere et al. 1989; Aldrich et al. 1989; Issa and Sullivan 1986; Collard et al. 1996) is the polysomnographic phenomenon of substantial increase in duration of REM sleep in a patient with untreated OSA when first undergoing CPAP titration. Similarly, some patients may exhibit an increase in the duration of slow wave sleep called slow wave sleep rebound (Brillante et al. 2012; Osuna et al. 2008; Verma et al. 2001).

REM rebound on the first night of CPAP use has multiple clinical implications. Clinically, patients exhibiting REM rebound have reported better sleep quality on CPAP titration night than on nights with untreated OSA (Osuna et al. 2008). In new CPAP users early CPAP adherence was found to be higher in patients exhibiting significant REM rebound during CPAP titration night (Koo et al. 2012). Currently, there are no consensus guidelines as to how much increase in duration of REM sleep in the titration night over the baseline sleep study (or baseline portion of a split night study) qualifies as REM rebound. The primary objective of this study was to determine if there is a statistically significant increase in percentage of REM sleep during CPAP titration as compared to baseline polysomnogram (PSG), in patients undergoing CPAP titration after being diagnosed with OSA. Secondary objectives were to quantify the relative increase in REM sleep duration and to predict polysomnographic factors associated with REM rebound. In order to meet the objectives of this review, a systematic review of the literature was performed to identify studies reporting REM rebound on the CPAP titration night as compared to the baseline sleep study night, and the quantitative data was used to determine the percent increase in REM sleep duration using a meta-analysis with random effects modeling.

\section{Methods}

The Preferred Reporting Items for Systematic Reviews and Meta-Analyses (PRISMA) statement checklist was used to report the findings of this systematic review (Fig. 1). Two authors (GN and MR) conducted a systematic search of electronic databases that included PubMed, Medline, Scopus, Web of Science and Cochrane Library from inception through March 12, 2017.

\section{Protocol}

The Tripler Army Medical Center Department of Clinical Investigation approved the protocol for this meta-analysis (Protocol TAMC 16N14).

\section{Search strategy}

The search included Medical Subject Headings (MeSH) terms, key words, and phrases in combinations to obviate missing articles due to the use of select terminology in the different databases. To make the search thorough, hand searches of the reference lists of relevant articles were performed in order to identify other pertinent articles. Also, meticulous grey literature and Google Scholar searches were performed to identify relevant publications that could have been missed during the electronic database search. Search was restricted to English language articles only. An example of a PubMed search is: ((("Continuous Positive Airway Pressure"[Mesh] AND "Sleep, REM"[Mesh] OR ("Continuous Positive Airway Pressure"[Mesh] AND "Sleep, REM"[Mesh]) AND "Sleep Apnea, Obstructive"[Mesh], Increase ${ }^{*}$ in REM sleep AND CPAP*, rapid eye movement sleep [tiab] AND CPAP [tiab]))) All articles were reviewed which discussed REM rebound in patients with OSA. Articles meeting the inclusion criteria were included in the systematic review.

Inclusion criteria using PICOS were: 1) Patients: those diagnosed with OSA, 2) Intervention: CPAP therapy, 3) Comparison: a) studies assessing amount of increase in REM sleep during CPAP titration as compared to amount of REM sleep in baseline PSG, b) and/or studies discussing slow-wave sleep rebound in addition to REM rebound, and c) studies looking for correlation between sleep quality or CPAP adherence and REM rebound, 4) Outcomes: the REM sleep duration differences on CPAP therapy during the titration night compared to the baseline PSG, and 5) Study design: randomized controlled trials, prospective and retrospective cohort studies, and case series. Exclusion criteria included: 1) Studies on sleep-disordered breathing (SDB) which exclusively discussed slow-wave sleep rebound, 2) Home sleep apnea studies with no electrooculography (EEG) and electromyography (EMG) monitoring capability, and 3) Individual case reports, editorials, review articles, and meeting abstracts.

\section{Statistical analysis}

Statistical evaluation was performed using Review Manager (REVMAN) Software version 5.3. The pre- and post-CPAP REM percentage, standard deviations (SD), mean differences (MD), 95\% confidence intervals (CI) and $p$-values were calculated using the IBM Statistical Package for Social Sciences (SPSS) software. Combined mean differences and 95\% CI were calculated only for studies reporting means and SD. The null hypothesis for the study was that there is no difference in percentages of REM sleep rebound between baseline PSG versus titration study and in order to test this hypothesis the data was analyzed using post minus pre CPAP therapy outcome data. A random effects modeling was utilized and the overall effect size estimation was performed by calculating the standardized mean difference (SMD). Cohen's guidelines were used to determine the magnitude of the effect size, and the SMD cutoff values were: 


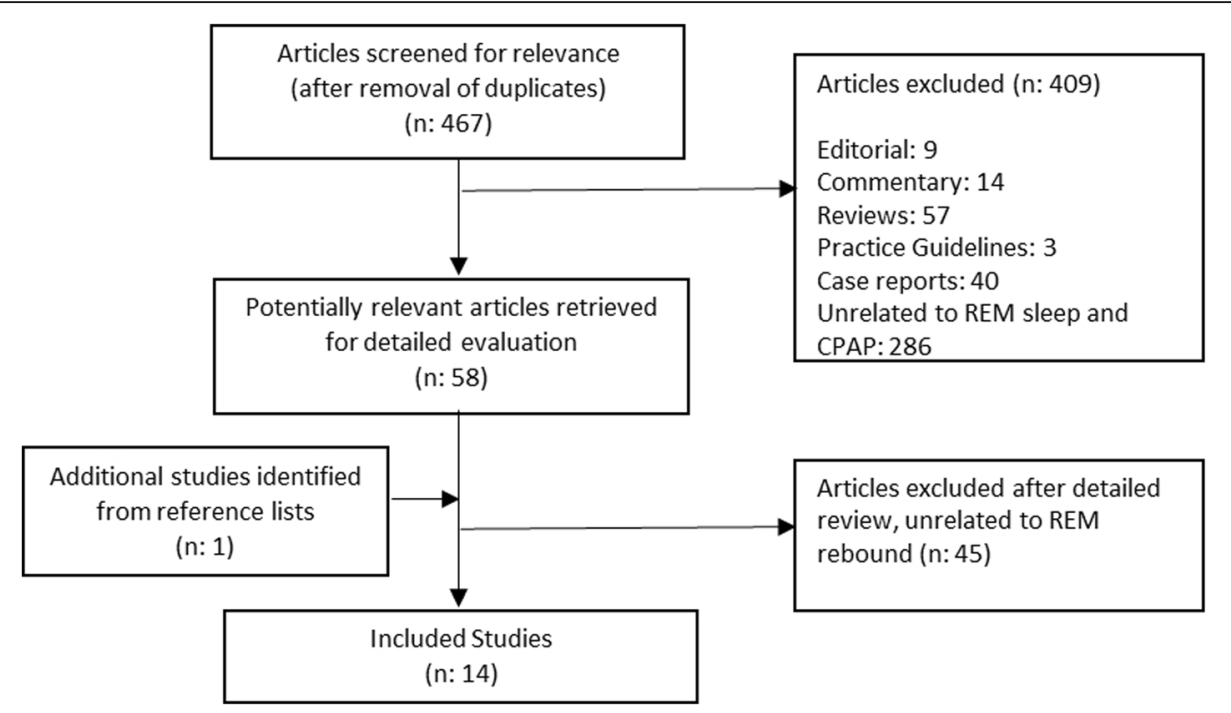

Fig. 1 Flowchart for study selection

small $=0.2$, medium $=0.5$ and large $=0.8($ Cohen 1988) . Heterogeneity was defined as a REVMAN Q-statistic value of $\leq 0.10$ (Lau et al. 1997), and the REVMAN I ${ }^{2}$ value cutoffs for inconsistency were $25 \%$ = low inconsistency, $50 \%=$ moderate inconsistency and $75 \%=$ high inconsistency (Higgins et al. 2003). The risk of bias was assessed (as recommended by the Cochrane Collaboration) by assessment of the funnel plots if there are at least ten studies in the variable of interest.

\section{Results}

Four hundred sixty-seven articles were screened, 58 were evaluated in full-text form, and 14 studies met the established criteria (Brillante et al. 2012; Koo et al. 2012; Kushida et al. 2011; Yaegashi et al. 2009; Osuna et al. 2008; Drake et al. 2003; Verma et al. 2001; Randerath et al. 2001; Parrino et al. 2000; Yamashiro and Kryger 1995; Lamphere et al. 1989; Aldrich et al. 1989; Issa and Sullivan 1986; Collard et al. 1996). Eleven of the 14 studies noted a statistically significant increase in amount of REM sleep during the titration night, compared to the baseline sleep study. Overall, data was reported for 1119 patients, with an average age $53.7 \pm 12.6$ years and body mass index (BMI) $34.6 \pm 11.2 \mathrm{~kg} / \mathrm{m}^{2}$ (Table 1). Patients with all severities of OSA were included with the highest mean apnea-hypopnea index (AHI) being $72.9 \pm 21.5$ in the Lamphere et al. study. All studies showed a predominance of male subjects, and they made up 66\% (Osuna et al.) to $95 \%$ (Collard et al.) of the patient population being studied.

Of the 14 studies meeting inclusion criteria, only three studies (Brillante, Koo and Osuna et al. studies) defined what they considered as REM rebound. The remaining studies provided some measure of increase in REM sleep during titration night compared to baseline sleep study. Most studies reported REM rebound in percentage increase in REM sleep duration when comparing titration to baseline sleep study except two studies (Aldrich et al. and Parrino et al.) where the relative increase in REM sleep was reported only in minutes. On an average, percentage of REM sleep in diagnostic PSG varied from 6.7 $\pm 9.3 \%$ in Koo et al. study to $18.4 \pm 2.0 \%$ in Issa et al. study. During the titration study, 11 of the 14 studies noted a statistically significant increase in amount of REM sleep compared to baseline sleep study, with two studies (Koo and Kushida et al.) not mentioning the statistically significant status. One study (Randerath et al.) reported that the increase was not statistically significant. REM sleep percentages during titration study varied between $17.1 \pm 7.4$ in Randerath et al. study to $30.6 \pm 2.0$ in Issa et al. study.

In studies reporting statistically significant increase in the percentage of REM sleep, the percentage of increase varied on average from $1.5 \%$ in Brillante et al. study to $14.1 \%$ in Yamashiro et al. study (Fig. 2). Although Koo et al. study reported an even higher increase in the average percentage of REM sleep at $16.6 \%$ (compared to Yamashiro study reporting an increase of $14.1 \%$ ) it was not stated whether this increase was statistically significant, when considering all patients. Incidentally, the two studies with highest percentage increase in REM sleep duration during CPAP titration also represented the only split night studies of this review.

As mentioned previously, three studies (Brillante, Koo and Osuna et al. studies) selected specific percentage cut-off values to define REM rebound. This was determined using a statistical prediction model used by the individual studies. Defining REM rebound as $20 \%$ or 


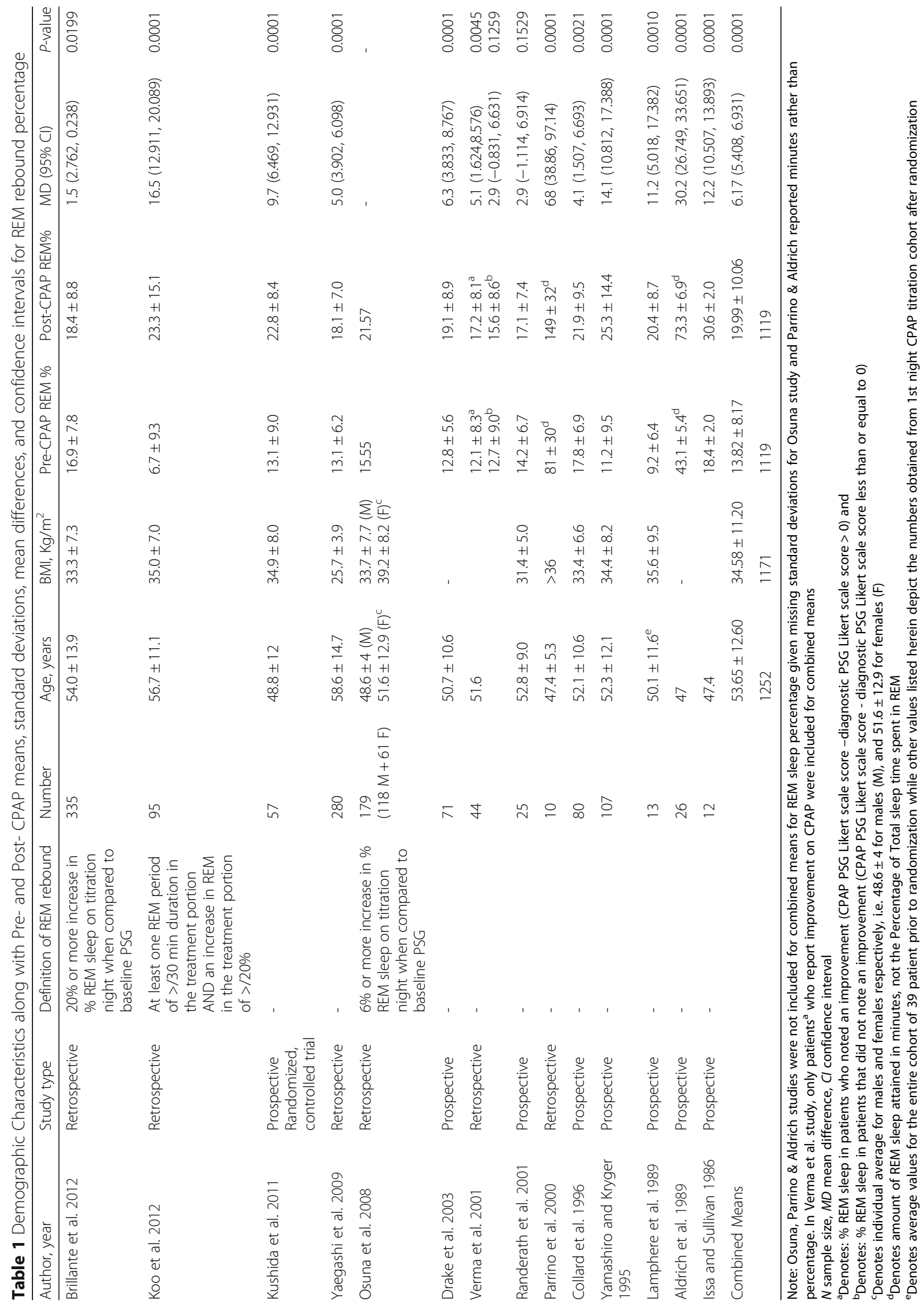




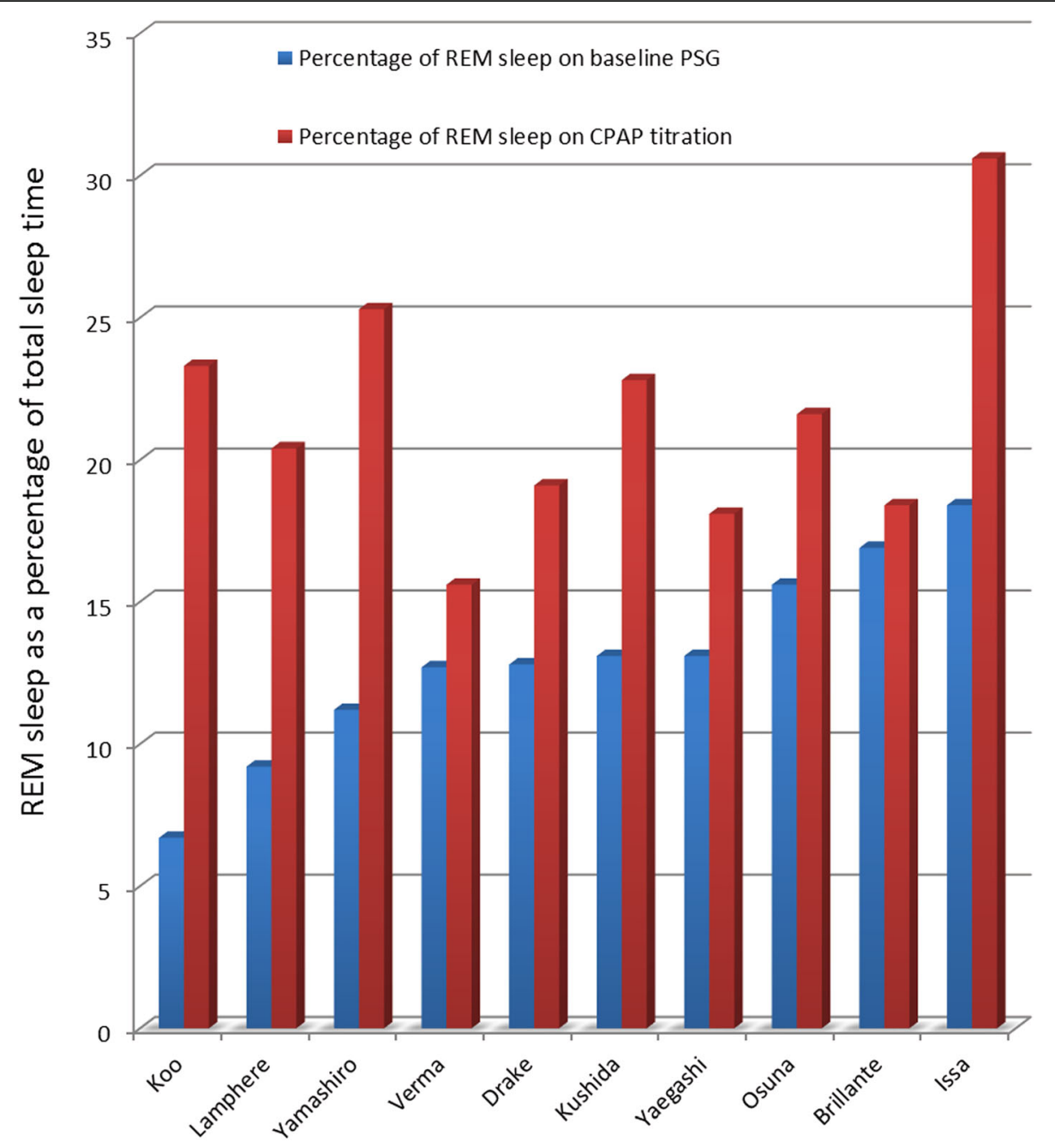

Study Author

Fig. 2 Bar graph comparing mean percentage of REM sleep attained on baseline PSG vs titration PSG for studies reporting a statistically significant increase in REM sleep

more increase in duration of REM sleep on titration night compared to baseline PSG, Brillante et al. and Koo et al. found that $23 \%$ and $37 \%$ of patients respectively, met criteria for REM rebound. On the other hand, employing $6 \%$ or more increase in REM sleep duration on titration night compared to baseline PSG as the definition of REM rebound, Osuna et al. found that $46 \%$ patients met criteria for REM rebound. All except two studies (Yamashiro et al. and Koo et al.) used data derived from titration studies to calculate REM rebound (Table 2).

\section{Mean differences and standardized mean differences}

Based on polysomnography data, the CPAP REM percentage means \pm standard deviations $(\mathrm{M} \pm \mathrm{SD})$ in 1119 patients increased from $13.8 \pm 8.2 \%$ to $20.0 \pm 10.1 \%$ (a mean difference of $6.2 \%$ ) which corresponds to a $44.6 \%$ relative percentage increase of REM sleep during the CPAP treatment compared to baseline (Table 1). Analysis of studies using random effects modeling was performed for REM rebound percentage on titration with mean difference (MD) 7.86\% [95\% CI 5.01, 10.70], overall effect $\mathrm{Z}=5.41, p$-value $<0.00001, \mathrm{Q}$ statistic $p$-value $<0.00001$ (significant heterogeneity), $\mathrm{I}^{2}=94 \%$ (high inconsistency) (Fig. 3). The mean difference of $7.9 \%$ corresponds to a $57 \%$ relative percentage increase of REM sleep during the CPAP treatment compared to baseline. The risk of bias was high as the funnel plot for REM rebound percentage mean difference was significantly asymmetrically shaped. The sensitivity analysis demonstrated two sub-groups of studies that were non-heterogenous when combined, the first non-heterogenous sub-group comprised the studies by Yaegashi et al., Drake et al., Verma et al., Randerath 
Table 2 REM rebound on CPAP titration: PSG parameters

\begin{tabular}{|c|c|c|c|}
\hline Study, year & Baseline AHI & $\begin{array}{l}\% \text { of patients with } \\
\text { REM rebound }\end{array}$ & $\begin{array}{l}\text { Titration (T) or Split } \\
\text { night (SN) study }\end{array}$ \\
\hline Brillante et al. 2012 & $40.7 \pm 26.1$ & $23 \%$ & $\mathrm{~T}$ \\
\hline Koo et al. 2012 & $44.5 \pm 28.8$ & $37 \%$ & SN \\
\hline Kushida et al. 2011 & $41.1 \pm 31.6$ & - & T \\
\hline Yaegashi et al. 2009 & $48.5 \pm 20.2$ & - & T \\
\hline Osuna et al. 2008 & $>10^{\mathrm{a}}$ & $46 \%$ & $\mathrm{~T}$ \\
\hline Drake et al. 2003 & $62.0 \pm 32.2$ & - & $\mathrm{T}$ \\
\hline Verma et al. 2001 & $\mathrm{RDI}>10^{\mathrm{a}}$ & - & T \\
\hline Randerath et al. 2001 & $32.2 \pm 18.1$ & - & $T^{c}$ \\
\hline Parrino et al. 2000 & 67.9 & - & T \\
\hline Collard et al. 1996 & $24.2 \pm 18.8$ & - & $\mathrm{T}$ \\
\hline Yamashiro and Kryger 1995 & $23.6 \pm 26.3$ & - & $S N^{b}$ \\
\hline Lamphere et al. 1989 & $72.9 \pm 21.5$ & - & T \\
\hline Aldrich et al. 1989 & $69.6 \pm 5.9$ & & T \\
\hline Issa and Sullivan 1986 & 57.6 & - & $\mathrm{T}$ \\
\hline
\end{tabular}

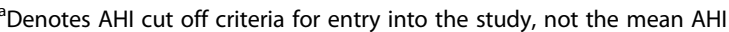

${ }^{b}$ Denotes that this study reported data from both Split night study as well as titration night study, but data tabulated represents Split night values as this was the study with first exposure to CPAP occurred and first REM rebound was witnessed

'Denotes PSG data collected from fixed CPAP setting, obtained using a manual CPAP titration 1-2 days prior

et al. and Collard et al. (Q-statistic $p=0.62$ and $\mathrm{I}^{2}=0 \%$ ); while the second sub-group comprised the studies by Yamashiro et al., Lampere et al. and Issa et al. (Q-statistic $p=0.54$ and $\mathrm{I}^{2}=0 \%$ ). The SMD is 0.90 [95\% CI 0.59 , 1.22], representing a large magnitude of effect (Cohen's guidelines) with an overall effect $\mathrm{Z}=5.60$, $p$-value $<0.00001$, Q statistic $p$-value $<0.00001$ (significant heterogeneity), $\mathrm{I}^{2}=90 \%$ (high inconsistency).

\section{Predictive factors for REM rebound}

Of the 14 studies, five studies (Brillante et al., Koo et al., Osuna et al., Verma et al. and Aldrich et al.) discussed predictive factors for REM rebound on CPAP titration (Table 3). High AHI on baseline PSG was found to be a predictive factor in all five studies, however a direct linear correlation could not be conclusively established between baseline AHI and "Delta REM" (denoting difference between percentage of REM sleep in post CPAP study and percentage of REM sleep in pre CPAP study), when data from all studies reporting REM sleep percentage were included (Fig. 4). Inability to establish direct correlation could be related to variability in rapidity of up-titration of individual studies as well as exclusion of Verma et al. and Aldrich et al. studies from scatter dot diagram data due to computing limitations; Aldrich et al. reported REM sleep in minutes rather than as percentages and in Verma et al. study, only patients who reported improvement on CPAP were included for combined means. Brillante et al. study also noted male gender and high arousal index on baseline PSG to be associated with REM rebound. A low percentage of REM sleep on baseline PSG was likely to result in greater "Delta REM" or REM rebound as found in three studies (Brillante et al., Koo et al. and Verma et al.). It was further corroborated when this inverse relationship was depicted on the scatter dot diagram when data from all

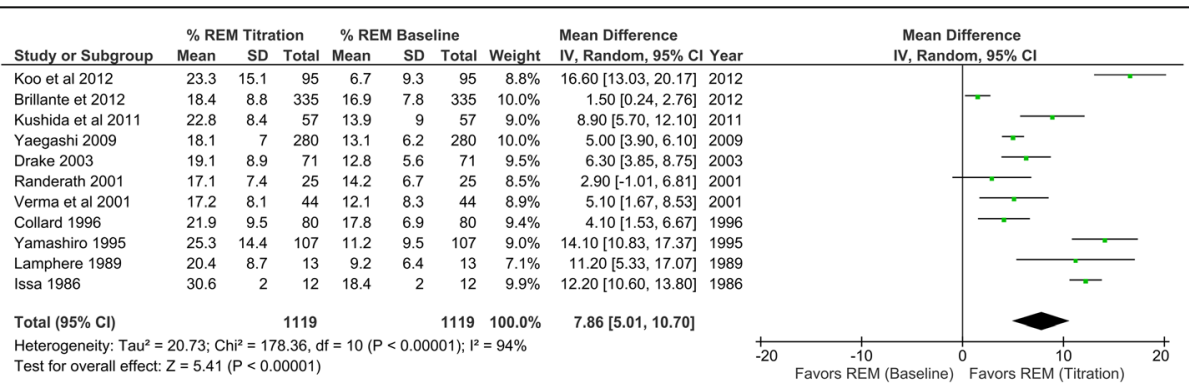

Fig. 3 Pre- and post-nasal CPAP REM Rebound (percent of REM sleep duration percentage) mean difference. (SD, standard deviation; Cl, confidence interval; REM, rapid eye movement sleep) 
Table 3 Predictive factors for REM rebound on CPAP titration

\begin{tabular}{|c|c|c|c|c|c|c|}
\hline Study & $\begin{array}{l}\text { High AHI on } \\
\text { baseline PSG }\end{array}$ & $\begin{array}{l}\text { Low } \% \text { of REM sleep } \\
\text { on baseline PSG }\end{array}$ & $\begin{array}{l}\text { High arousal index } \\
\text { on baseline PSG }\end{array}$ & Male gender & $\begin{array}{l}\text { Low oxygen saturation } \\
\text { on baseline PSG }\end{array}$ & High BMI \\
\hline Brillante et al. & $\checkmark$ & $\checkmark$ & $\checkmark$ & $\checkmark$ & - & $x$ \\
\hline Koo et al. & $\checkmark$ & - & - & - & - & - \\
\hline Osuna et al. & $\checkmark$ & $\checkmark$ & - & - & - & $\checkmark$ \\
\hline Verma et al. & $\checkmark$ & - & - & - & - & - \\
\hline Aldrich et al. & $\checkmark$ & $\checkmark$ & - & - & $\checkmark$ & - \\
\hline
\end{tabular}

$\checkmark$ : Denotes that the parameter was studied and was found to be statistically significant factor for REM rebound

X: Denotes that the parameter was studied but was not found to be statistically significant factor for REM rebound

- : Denotes that the parameter was not studied

studies reporting REM sleep percentage were included (Fig. 5). In the study by Osuna et al. (average BMI: $35.5 \mathrm{~kg} / \mathrm{m}^{2}$ ), obesity was associated with REM rebound; however, the study by Brillante et al. (average BMI: $33.3 \mathrm{~kg} / \mathrm{m}^{2}$ ) failed to find a statistically significant association between body weight and REM rebound. Low oxygen saturation on baseline PSG was associated with REM rebound as noted by Aldrich et al.

\section{Study quality assessment}

Overall, the studies included in this review met between four to eight out of a total of eight quality assessment parameters, with most studies meeting five to six parameters. The quality of studies per "NICE guidelines" is outlined in Table 4.

\section{Discussion}

This systematic review and meta-analysis has five main findings. First, eleven of fourteen studies reported a statistically significant increase in REM sleep duration during titration night compared the baseline sleep study. While all studies report REM rebound, only a few studies define what REM rebound entails based on their statistical prediction models. Studies adopted cut-off

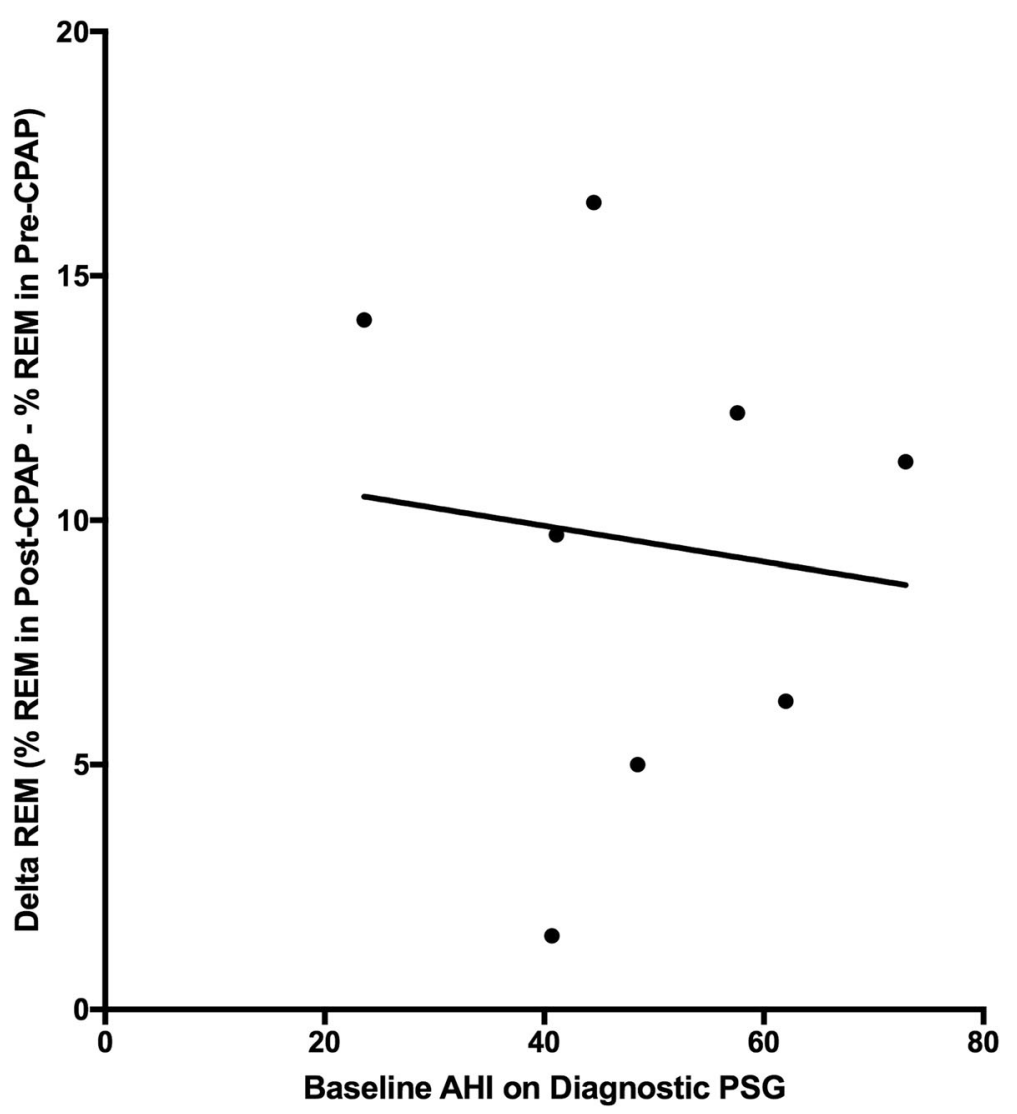

Fig. 4 Correlation between AHI on baseline PSG and amount of REM rebound (Delta REM) on CPAP titration 


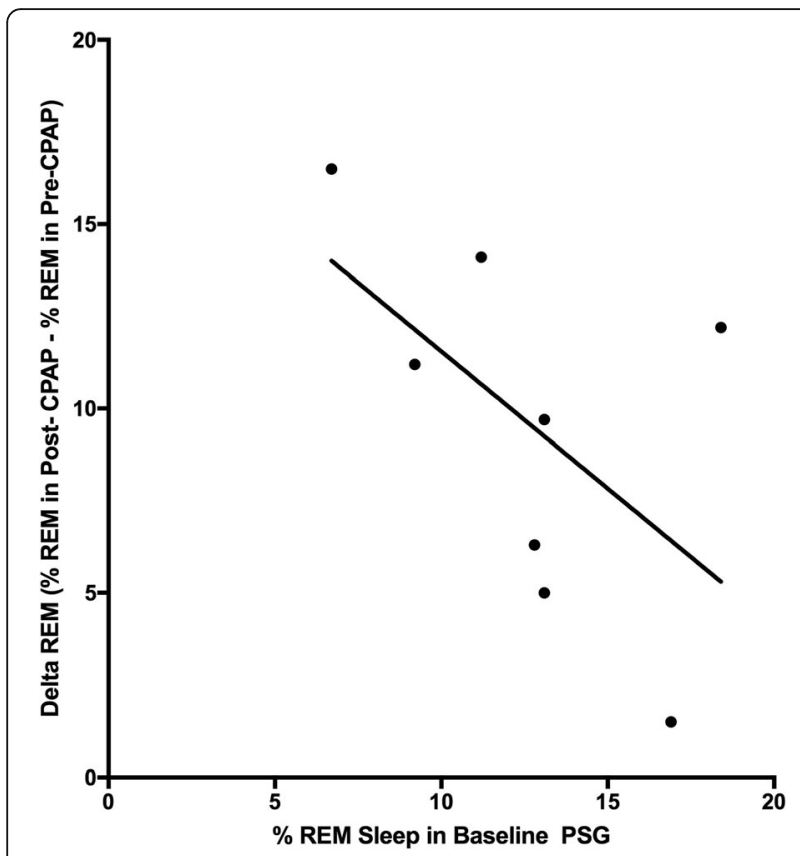

Fig. $\mathbf{5}$ Correlation between percentage of REM sleep on baseline PSG and amount of REM rebound (Delta REM) on CPAP titration

Table 4 Assessment of *quality of studies included in the systematic review

\begin{tabular}{lllllllll}
\hline Study author & 1 & 2 & 3 & 4 & 5 & 6 & 7 & 8 \\
\hline Brillante et al. & No & Yes & Yes & Yes & No & Yes & Yes & Yes \\
Koo et al. & No & Yes & Yes & Yes & No & No & Yes & Yes \\
Kushida et al. & Yes & Yes & Yes & Yes & Yes & Yes & Yes & Yes \\
Yaegashi et al. & No & Yes & Yes & Yes & No & Yes & Yes & Yes \\
Osuna et al. & No & Yes & No & Yes & No & Yes & Yes & Yes \\
Drake et al. & No & Yes & Yes & Yes & Yes & Yes & Yes & Yes \\
Verma et al. & No & Yes & Yes & Yes & No & Yes & Yes & Yes \\
Randerath et al. & No & Yes & Yes & Yes & Yes & Yes & Yes & Yes \\
Parrino et al. & No & Yes & No & Yes & No & No & Yes & Yes \\
Collard et al. & No & Yes & No & Yes & Yes & Yes & Yes & Yes \\
Yamashiro et al. & No & Yes & Yes & Yes & Yes & No & Yes & Yes \\
Lamphere et al. & No & Yes & No & Yes & Yes & No & Yes & Yes \\
Aldrich et al. & No & Yes & Yes & Yes & Yes & Yes & Yes & Yes \\
Issa et al. & No & No & No & Yes & Yes & No & Yes & Yes \\
\hline
\end{tabular}

${ }^{*}$ Quality assessment of the included studies checklist from questions from National Institute for Health and Clinical Excellence (NICE) 1-8:

1) Case series collected in more than one center?

2) Is the hypothesis/aim/objective of the study clearly described?

3) Are the inclusion and exclusion criteria clearly reported?

4) Is there a clear definition of the outcomes reported?

5) Were data collected prospectively?

6) Is there an explicit statement that patients were recruited consecutively?

7) Are the main findings of the study clearly described?

8) Are outcomes stratified? criteria of 6\%-20\% increase in percentage REM sleep on titration night when compared to baseline PSG to define REM rebound. Accepting a lower REM percentage increase as the cut -off criteria for REM rebound (when comparing REM percentage in titration study to REM percentage in baseline PSG study) leads to reporting a higher prevalence of REM rebound on CPAP titration. Accordingly, based on cut-off criteria used to define REM rebound, the prevalence of REM rebound varies between 23 and $46 \%$ in all patients undergoing CPAP titration study.

Second, when the specific cut-off criteria (in terms of duration of increase in REM sleep percentage) defining REM rebound are overlooked, the majority of patients undergoing a CPAP titration experience increased duration of REM sleep compared to their baseline sleep study. The percentage increase in REM sleep duration varies between 1.5 and $14 \%$ and may depend on certain polysomnographic and demographic factors. The mean difference of $7.86 \%$ corresponds to a $57 \%$ relative percentage increase of REM sleep during the CPAP treatment night compared to the baseline study. When evaluating the effect size based on Cohen's guidelines, the SMD is 0.90 [95\% CI $0.59,1.22]$, which is defined as a large effect. Interestingly, the sensitivity analysis demonstrated two sub-groups of studies that were non-heterogenous when combined, and the subgroups tended to be grouped by the year of the study publication; with the first non-heterogenous subgroup consisted of studies from 1996 to 2009 (Yaegashi et al., Drake et al., Verma et al., Randerath et al. and Collard et al.) with a Q-statistic $p=0.62$ and $\mathrm{I}^{2}=0 \%$. The second non-heterogenous sub-group consisted of studies from 1986 to 1995 (Yamashiro et al., Lampere et al. and Issa et al.) with a Q-statistic $p=0.54$ and $\mathrm{I}^{2}=0 \%$. It is unclear as to why these two sub-groups were nonheterogenous when grouped alone, but did cause heterogeneity and inconsistency when grouped as part of the fourteen studies.

Third, a high percentage increase in REM sleep was observed specifically during split night studies. A relatively high proportion of REM sleep during split night studies (compared to full night titration studies) could be multifactorial, associated with at least three factors: (A) the natural timing of REM sleep, (B) the rapidity of up-titrations and $(C)$ the baseline severity of OSA. First, the PAP titration portion of split night study temporally coincides with timing for natural preponderance of REM sleep in the latter half of sleep period (Ciftci et al. 2008). Second, in contrast to dedicated, full night titration studies, during split-night studies, sleep technicians might be up-titrating the pressures relatively rapidly due to limited titration window leading to early, brief but repetitive bursts of saw tooth waves or low voltage mixed frequency REM spikes. Lastly, patients who 
undergo split night study typically have severe OSA. In such patients both high baseline AHI and high arousal index from sleep fragmentation, are being simultaneously resolved with PAP therapy, which may be promoting additional periods of restorative REM sleep. Immediate increased REM duration from CPAP use as early as after one night have been observed (Loredo et al. 2006; Issa and Sullivan 1986). So, it is plausible that the rebound REM increase occurs even within a few hours of initiation of CPAP, given that untreated OSA represents a state of several years of chronic partial sleep deprivation as well as sleep fragmentation.

We speculate that the quantity of REM rebound could depend upon rapidity of up- titrations as well how quickly the patient adapted to these up-titrations. In some studies, rapid CPAP up-titration may have led to early attainment of therapeutic pressure providing more time during sleep period to exhibit REM. In other studies, a more conservative and cautious approach may have lead to at least some REM sleep attained chronologically prior to attainment of therapeutic CPAP pressure leading to less REM rebound. Of note, about 5-20\% patients may have experienced treatment emergent central sleep apnea or TECSA on first exposure to CPAP during these studies (Nigam et al. 2016). TECSA may have lead to increased arousal index and sleep fragmentation thereby limiting REM rebound which would be subsequently seen after several weeks of CPAP use in this cohort of CPAP users.

Fourth, certain polysomnographic and demographic factors could enhance REM rebound. Two such factors predicting enhanced Delta REM (and thereby predicting substantial REM rebound) as suggested in several studies include: 1) a lower amount of REM sleep on diagnostic PSG (strong association) and 2) a higher severity of OSA as noted by the AHI during diagnostic PSG (weak association, if any). Other possible associations include higher arousal index on baseline PSG, low oxygen saturation on baseline PSG, male gender, higher BMI and utilization of split-night PSG to study REM rebound. Additional research is needed to further evaluate the variables that predict which patients are more likely to experience REM rebound.

Fifth, almost half of the currently published studies were retrospective observational studies and prone to limitations of any retrospective study. There were differences amongst studies as to the definition of REM rebound. The sleep stage and respiratory events scoring rules have undergone considerable changes over the last few decades. Given the review includes studies conducted over last 30 years, the majority of the included studies were scored using Rechtschaffen and Kales manual for the scoring of sleep stages, while others were scored based on the American Academy of Sleep
Medicine scoring manual introduced in 2007. The rules for scoring respiratory events have undergone considerable changes as well over the years, making it difficult to directly compare baseline AHIs reported by different authors; however, given that REM is defined based on the electroencephalogram, we believe that despite the differences in the AHI scoring criteria, the percentage of sleep scored for each of the sleep stages, including REM, should not change.

\section{Limitations}

We acknowledge that the expression "percentage" increase in REM sleep could arithmetically, sometimes get misconstrued as demonstrated by the following example: a doubling of REM sleep time from 5 to $10 \mathrm{~min}$ is not the same as a doubling of REM sleep time from 20 to $40 \mathrm{~min}$. Therefore, we suggest that the percentage of relative increase in REM sleep should be interpreted cautiously in appropriate clinical and polysomnographic context. This "percentage" increase in REM sleep was chosen as they are conventional mathematical tools for data representation. Also, percentages are employed as a standard practice for reporting the proportion of the time spent in different sleep stages on a polysomnogram report. Unfortunately, as demonstrated by the above example, even a small amount of additional time spent in REM during the treatment night could translate to a significantly inflated percentage increase in REM sleep. Consistent with all systematic reviews, it is possible that we missed identifying studies that met our criteria; however, to decrease this possibility, we had two authors search independently. Additionally, our search was restricted to English-language manuscripts only, and there may have been additional studies in other languages. Lastly, as demonstrated by the funnel plot, there is a high risk of publication bias, therefore, it is possible that researchers with negative findings either never shared their findings, or those inferences may have been rejected after submission. Previous studies suggest that sleep stages (including REM stage) recovery after CPAP titration is not an instant "all-or-none phenomenon" but requires several days to weeks before complete return to normal control values (Parrino et al. 2005). This might have lead to underestimation of REM rebound as sleep reorganization is a dynamic adaptive process spanning several weeks, and our work only captured the initial phases of effective CPAP treatment. Our analysis was restricted to REM rebound in terms of duration and percentages; it did not include analysis of REM latency as this parameter was not recorded and shared by most constituent studies.

\section{Conclusion}

Significant increase in REM sleep duration can occur in the first CPAP titration night compared to REM sleep 
duration noted on the baseline sleep study. In studies reporting REM rebound, the REM sleep duration increased by $57 \%$ during the first CPAP titration night compared to the baseline sleep study. Although no consensus guidelines exist, various retrospective studies have outlined that $6-20 \%$ increase in percentage of REM sleep on titration night compared to baseline PSG could be indicative of REM rebound. The most crucial polysomnographic factor that predicts and promotes REM rebound is low amount of REM sleep attained on the diagnostic PSG. In order to increase the level of evidence, additional high quality, prospective studies will be required in the future.

\footnotetext{
Abbreviations

AHI: Apnea-hypopnea index; BMI: Body mass index; Cl: Confidence interval; CPAP: Continuous positive airway pressure; EEG: Electrooculography; EMG: Electromyography; MD: Mean differences; MeSH: Medical Subject Headings; NREM: Non-rapid eye movement; OSA: Obstructive sleep apnea; PICOS: Patients, Intervention, Comparison, Outcomes, Study Design; PRISMA: Preferred Reporting Items for Systematic Reviews and MetaAnalyses; PSG: Polysomnogram; REM: Rapid eye movement; REVMAN: Review Manager; SD: Standard deviations; SDB: Sleep disorderd breathing; SMD: Standardized mean differences; SPSS: Statistical Package for Social Sciences
}

\section{Acknowledgements}

None.

\section{Funding}

None.

\section{Availability of data and materials}

Not applicable.

\section{Authors' contributions}

GN: Substantial contributions to the conception or design of the work; the acquisition, analysis, and interpretation of data for the work; AND Drafting and revising it critically for important intellectual content; AND Final approval of the version to be published; AND Agreement to be accountable for all aspects of the work in ensuring that questions related to the accuracy or integrity of any part of the work are appropriately investigated and resolved. MR: Substantial contributions to the conception of the work; the acquisition, analysis, and interpretation of data for the work; AND Drafting the work and revising it critically for important intellectual content; AND Final approval of the version to be published; AND Agreement to be accountable for all aspects of the work in ensuring that questions related to the accuracy or integrity of any part of the work are appropriately investigated and resolved. MC : Substantial contributions to the interpretation of data for the work; AND revising it critically for important intellectual content; AND Final approval of the version to be published; AND Agreement to be accountable for all aspects of the work in ensuring that questions related to the accuracy or integrity of any part of the work are appropriately investigated and resolved.

\section{Authors' information}

None.

\section{Competing interests}

The authors declare that they have no competing interests. Authors do not have anything to disclose, this study was not supported by industry. The views expressed in this manuscript are those of the authors and do not reflect the official policy or position of the Department of the Army, Department of Defense, or the US Government.

\section{Consent for publication}

Not applicable.

\section{Ethics approval and consent to participate}

The Tripler Army Medical Center Department of Clinical Investigation approved the protocol for this meta-analysis (Protocol TAMC 16 N14).

\section{Publisher's Note}

Springer Nature remains neutral with regard to jurisdictional claims in published maps and institutional affiliations.

\section{Author details}

${ }^{1}$ Clay County Hospital, 911 Stacy Burk Drive, Flora, IL 62839, USA. ${ }^{2}$ Division of Otolaryngology, Sleep Surgery, and Sleep Medicine, Tripler Army Medical Center, 1 Jarrett White Road, Tripler AMC, Honolulu, HI 96859, USA. ${ }^{3}$ Sunnyside Community Hospital \& Clinics, 208 N. Euclid, Grandview, WA 98930, USA

Received: 15 March 2017 Accepted: 16 May 2017

Published online: 26 May 2017

\section{References}

Agnew Jr HW, Webb WB, Williams RL. Comparison of stage four and 1-rem sleep deprivation. Percept Mot Skills. 1967;24(3):851-8.

Aldrich M, Eiser A, Lee M, Shipley JE. Effects of continuous positive airway pressure on phasic events of REM sleep in patients with obstructive sleep apnea. Sleep. 1989;12(5):413-9.

Brillante R, Cossa G, Liu PY, Laks L. Rapid eye movement and slow-wave sleep rebound after one night of continuous positive airway pressure for obstructive sleep apnoea. Respirology. 2012;17(3):547-53.

Ciftci B, Ciftci TU, Guven SF. Split-night versus full-night polysomnography: comparison of the first and second parts of the night. Arch Bronconeumol. 2008:44(1):3-7.

Cohen J. Statistical power analysis for the behavioral sciences. Kawrence Erlbaum Associates: Hillsdale; 1988

Collard P, Dury M, Delguste P, Aubert G, Rodenstein DO. Movement arousals and sleep-related disordered breathing in adults. Am J Respir Crit Care Med. 1996;154(2 Pt 1):454-9.

Drake CL, Day R, Hudgel D, Stefadu Y, Parks M, Syron ML, et al. Sleep during titration predicts continuous positive airway pressure compliance. Sleep. 2003;26(3):308-11.

Higgins JP, Thompson SG, Deeks JJ, Altman DG. Measuring inconsistency in meta-analyses. BMJ. 2003;327(7414):557-60.

Issa FG, Sullivan CE. The immediate effects of nasal continuous positive airway pressure treatment on sleep pattern in patients with obstructive sleep apnea syndrome. Electroencephalogr Clin Neurophysiol. 1986;63(1):10-7.

Koo BB, Wiggins R, Molina C. REM rebound and CPAP compliance. Sleep Med. 2012:13(7):864-8.

Kushida CA, Berry RB, Blau A, Crabtree T, Fietze I, Kryger MH, et al. Positive airway pressure initiation: a randomized controlled trial to assess the impact of therapy mode and titration process on efficacy, adherence, and outcomes. Sleep. 2011;34(8):1083-92.

Lamphere J, Roehrs T, Wittig R, Zorick F, Conway WA, Roth T. Recovery of alertness after CPAP in apnea. Chest. 1989:96(6):1364-7.

Lau J, loannidis JP, Schmid CH. Quantitative synthesis in systematic reviews. Ann Intern Med. 1997;127(9):820-6.

Loredo JS, Ancoli-Israel S, Kim EJ, Lim WJ, Dimsdale JE. Effect of continuous positive airway pressure versus supplemental oxygen on sleep quality in obstructive sleep apnea: a placebo-CPAP-controlled study. Sleep. 2006;29(4):564-71.

Nigam G, Pathak C, Riaz M. A systematic review on prevalence and risk factors associated with treatment- emergent central sleep apnea. Ann Thorac Med. 2016;11(3):202-10.

Osuna S, Siddiqui F, Vanegas MA, Walters AS, Chokroverty S. Prevalence and factors affecting REM and slow wave sleep rebound on CPAP titration study in patients with obstructive sleep apnea. Revista de la Facultad de Medicina. 2008;56(1):4-10.

Parrino L, Smerieri A, Boselli M, Spaggiari MC, Terzano MG. Sleep reactivity during acute nasal CPAP in obstructive sleep apnea syndrome. Neurology. 2000:54(8):1633-40.

Parrino L, Thomas RJ, Smerieri A, Spaggiari MC, Del Felice A, Terzano MG. Reorganization of sleep patterns in severe OSAS under prolonged CPAP treatment. Clin Neurophysiol. 2005;116(9):2228-39. 
Randerath WJ, Galetke W, David M, Siebrecht H, Sanner B, Ruhle K. Prospective randomized comparison of impedance-controlled auto-continuous positive airway pressure (APAP(FOT)) with constant CPAP. Sleep Med. 2001;2(2):115-24. Remmers JE, De Groot WJ, Sauerland EK, Anch AM. Pathogenesis of upper airway occlusion during sleep. J Appl Physiol Respir Environ Exerc Physiol. 1978:44(6):931-8.

Verma A, Radtke RA, VanLandingham KE, King JH, Husain AM. Slow wave sleep rebound and REM rebound following the first night of treatment with CPAP for sleep apnea: correlation with subjective improvement in sleep quality. Sleep Med. 2001;2(3):215-23.

Yaegashi H, Fujimoto K, Abe H, Orii K, Eda S, Kubo K. Characteristics of Japanese patients with complex sleep apnea syndrome: a retrospective comparison with obstructive sleep apnea syndrome. Intern Med. 2009;48(6):427-32.

Yamashiro Y, Kryger MH. CPAP titration for sleep apnea using a split-night protocol. Chest. 1995;107(1):62-6.

Submit your next manuscript to BioMed Central and we will help you at every step:

- We accept pre-submission inquiries

- Our selector tool helps you to find the most relevant journal

- We provide round the clock customer support

- Convenient online submission

- Thorough peer review

- Inclusion in PubMed and all major indexing services

- Maximum visibility for your research

Submit your manuscript at www.biomedcentral.com/submit
Biomed Central 